\title{
Structural analysis, band gap tuning and photoluminescence properties of Fe-doped $\mathrm{TiO}_{2}$ nanoparticles
}

\author{
Tinku Ali, Ateeq Ahmed and P.Tripathi*
}

Department of Applied Physics, Aligarh Muslim University, Aligarh-202002, U.P, India Centre of Excellence in Materials Science (Nanomaterials), Department of Applied Physics, Faculty of Engineering \& Technology, Aligarh Muslim University, Aligarh 202002, India

*Email: pushpendratripathi05@gmail.com

We have studied the structural and optical properties of $\mathrm{Fe}(0,3,5,7 \& 10 \mathrm{~mol} \%)$ doped $\mathrm{TiO}_{2}$ (titania) nanoparticles (NPs) synthesized by employing acid modified sol-gel method. Structural and optical properties were examined by using different characterization techniques such as X-ray diffraction (XRD), Scanning electron microscope (SEM), Transmission electron microscope (TEM), UV-visible spectroscopy, Fourier-transform Infrared (FTIR) spectroscopy and photoluminescence (PL).

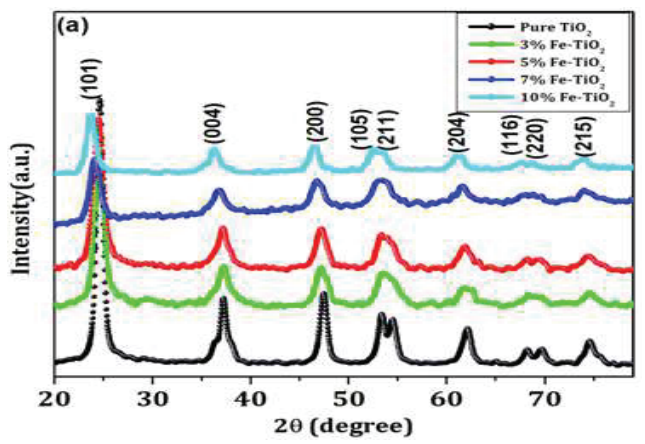

Figure 1: The XRD patterns of the Fe-doped $\mathrm{TiO}_{2}$

The powder XRD spectra as shown in Figure 1 reveal that the particles are in pure tetragonal anatase phase structure. The average crystallite size of all samples was estimated by using Debye Scherrer's formula. Surface morphology and elemental analysis were carried out by SEM, TEM and EDAX analysis. FTIR spectra show the strong bonding interactions of $\mathrm{Fe}$ ions with the $\mathrm{TiO}_{2}$ lattice. The UV-visible and PL spectra as shown in Figure 2 demonstrated an absorption shift in $\mathrm{Fe}$-doped $\mathrm{TiO}_{2}$ nanoparticles to have longer wavelengths. This study can provide a new insight for constructing the photocatalysts, which can be used in environmental pollution and water treatment applications.

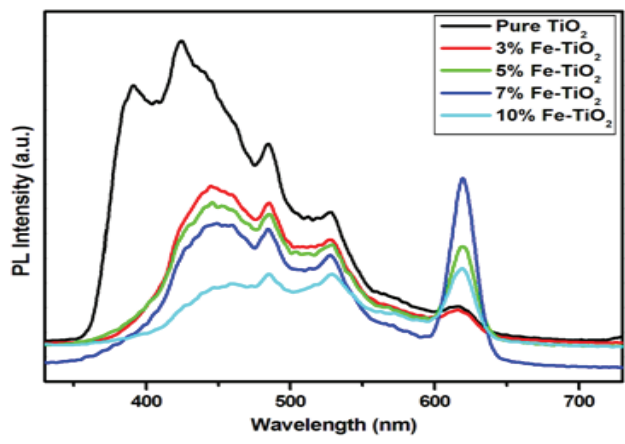

Figure 2: Photoluminescence spectra of pure and Fe doped $\mathrm{TiO}_{2}$ NPs

\section{References}

1. J. O. Carneiro, V. Teixeira, A. Portinha et al., Materials Science and Engineering B (2007) 144150 .

2. H. Lachheb, E. Puzenat, A. Houas et al., Applied Catalysis B. 39 (2002) 75-90. 